\title{
Una mirada al departamento de Carazo desde los estudios regionales
}

\author{
M.Sc. Wilmer Martín Guevara \\ Máster en Didácticas de la Ciencias Sociales \\ UNAN-Managua, FAREM-Carazo \\ wilunan2016@gmail.com
}

Fecha de recepción: 22 de octubre, 2019 / Fecha de aceptación: 12 de noviembre, 2019

https://doi.org/10.5377/torreon.v8i21.8855

Palabras clave: región histórica, etnografía, ecología cultural, integración y articulación.

\section{RESUMEN}

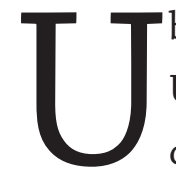

bicado en la reciente historiografía regional nicaragüense, impulsada por la Universidad Nacional Autónoma de Nicaragua, Managua (UNAN-Managua) desde la década de los 80 , el presente artículo $^{1}$ se propone demostrar que las regiones históricas tienen dinámicas de integración y articulación que no necesariamente se corresponden con las divisiones políticas administrativas del territorio. Para explicar esta dinámica se ha recurrido al método de la ecología cultural del antropólogo Julian Steward (1955) que propone entender las regiones desde su núcleo cultural, es decir, la adaptación e interacción del hombre con su entorno; por supuesto, el trabajo etnográfico ha sido fundamental. Los resultados revelan que Carazo, tiene cuatro regiones históricas internas. Por lo cual se pudo concluir, entre otras cosas que tanto la divisiones políticas como regionales coexisten sin 1. Este artículo nace de la tesis doctoral "Identidades en movimiento: café, cultura y turismo en el Triángulo de oro del café, en las postrimerías del siglo XIX e inicios del siglo XXI", que se inscribe en la línea de investigación "Los procesos de conformación regional: historia, actualidad y perspectiva". El programa de doctorado en Historia con mención en Estudios Regionales y Locales Transdisciplinario (2014-2019) está bajo la dirección de la Facultad de Humanidades y Ciencias Jurídicas de la Universidad Nacional Autónoma de Nicaragua, Managua (UNANManagua). El estudio doctoral cuenta con el 50\% de financiamiento de la Facultad Regional Multidisciplinaria de Carazo, FAREM-Carazo de la UNAN-Managua. 
oponerse una a la otra, pero se destaca el entendimiento regional como una posibilidad de acortar la brecha centro-periferia.

\section{INTRODUCCIÓN}

El Municipio es la unidad base de la división política administrativa del país y debe tomar en cuenta la identidad natural, socio-económica y cultural de la población que lo integra. Así se establece en la ley de los municipios en Nicaragua. Sin embargo, las poblaciones tienen una dinámica muy distinta en su interacción. Los gobiernos municipales por su parte, solo pueden atender parcialmente las necesidades de sus habitantes, priorizando por lo general, a las poblaciones urbanas y marginando -muchas veces sin las intenciones de hacerlo- a las poblaciones periféricas. Los municipios se convierten en islas que deben resolver solos sus problemas de producción, acceso a los recursos o tecnologías pertinentes para realidades concretas, etc., cuando pudieran ser más eficaces en su gestión si abordaran algunos problemas de manera conjunta. En el presente estudio se presenta una regionalización del territorio departamental que atiende a las particularidades de las poblaciones y no de las líneas imaginarias que atraviesan los dominios de la municipalidad. En este sentido se han propuesto dos objetivos específicos: a) identificar la organización regional del departamento de Carazo, y b) explicar la interacción y articulación de dichas regiones sin menoscabo de las fronteras internas que dividen al departamento.

\section{METODOLOGÍA}

Debe quedar claro que no existe un método regional de estudio, pero sí una estrategia de investigación, y eso es el enfoque regional. El método de esta investigación es la ecología cultural desarrollada y acuñada en 1955 por el antropólogo y arqueólogo norteamericano Julián H. Steward (1902-1972) que niega el enfoque determinista y unilineal de la evolución, para explicar el cambio cultural desde el interaccionismo y la multilinealidad. Su método comprende tres procedimientos fundamentales 1) Analizar la interrelación entre las tecnologías de explotación y el entorno (medio ambiente), 2) Analizar los patrones de conducta involucrados en la explotación y tecnología de explotación 3) Determinar cómo los modelos de conducta afectan otro aspecto de la cultura, entendiendo que el núcleo de la cultura es la identidad. (Steward 1955: pp.37-38). El trabajo etnográfico fue fundamental, porque da vida al dato frio de las estadísticas o los estudios técnicos de las regiones, especialmente porque estas se consideran como espacios vividos. En este sentido fue importante la utilización de la entrevista y la observación participante.

\section{La región como proceso}

El concepto de región ha sido muy debatido desde diferentes enfoques y disciplinas, sin que se haya logrado hasta el momento un consenso. Se realizará una revisión, seguramente de forma inacabada, a través de otros autores, de las principales reflexiones que se ha realizado sobre este concepto desde la geografía económica, la historia regional y la antropología social. 
Un vistazo a la región desde la geografía: La región de los geógrafos en su tradición más profunda, es decir, la región natural, no se cuestionaba; estaba allí. Con el inicio de la regionalización, entendida desde la academia como el proceso de clasificación que conduce a una tipología (Espejo, 2003:71), la región debía tomar énfasis y allí comenzó la divergencia en los enfoques. Espejo Martín identifica dos corrientes de pensamiento; la de los positivistas, quienes la definieron como un ente real, en contraposición a la corriente de la geografía crítica, que la entiende como una construcción mental. Los primeros definen la región como realidad observable a varias escalas, organizada por grupos humanos y clasificable a partir de criterios como el clima, relieve, vegetación y cultivo, fauna, hidrografía y suelo. Estos elementos determinaban las formas de vida y ocupación de los grupos humanos que la habitaban. Los segundos, consideran a la región como una construcción mental; es decir una categoría de análisis que permite desde la óptica del investigador, elegir los criterios necesario para definirla a partir de sus objetivos, de modo que la región toma vida propia, en ese sentido, Molina (1986) dice que la región con ente único, no existe.

Por su parte, señala Bataillón en 1979 citado por Boehm (1997:22) que "son dos los asuntos que los geógrafos tratan; la conformación regional de la superficie terrestre y la huella del hombre sobre ella”. Esta visión material de la región, para Brigitte Boehm (2007), da preferencia a la economía para su explicación, y evoca a Bataillon (1979) y Bassol Batalla (1986) para explicar la construcción regional desde la geografía económica. El primero opta por la articulación histórica, que la define por su polarización y difusión en torno a las ciudades, en cambio el segundo prefiere el modelo de la integración histórica, que concibe a la región como el escenario de las luchas de clases a partir de la visión marxista, a pesar de esta divergencia, ambos reconocen el papel central del Estado en los procesos integradores.

En suma, desde los geógrafos positivistas hasta los humanistas, como lo ha planteado Molina (1986) la región se concibe como un ente externo, que podemos repartir, planificar, intervenir, en fin, modificar e incluso vivir, para la geografía económica por su parte por su parte, la región se construye por procesos de difusión y utilización (apropiación). En ambos casos, persiste una visión pasiva de la región.

La región de los historiadores regionales: la comprensión sobre la construcción y transformación del espacio de los geógrafos, se diluye cuando lo abordamos desde la historia regional. En la ponencia presentada en el año 2000 por la doctora Ivette García González en el IV taller internacional "problemas teóricos y prácticos de la historia regional y local” se refería al espacio regional como "una forma de organización de la múltiples relaciones sociales, de manera que siendo una totalidad social, toma un carácter de estructura subordinante-subordinada" (2000a:7). En palabras de Boehm (1997:26) se comienzan a ver en el espacio, no solo el paisaje y al humano, sino "las redes sociales, las relaciones políticas, los flujos económicos, los rasgos 
culturales, el alcance visual y efectivo de los habitantes (...) la región se amiba y sus límites se diluyen".

Como es evidente, el espacio también ha sido preocupación de la historia regional, sin embargo, el tiempo constituye su principal interés, pues no comparten con la historia tradicional la visión de "los hechos congelados en el tiempo", sino que los reinterpretan en sus movimientos evolutivos. El problema de la periodización en los estudios regionales es uno de los más importantes, pues según la doctora cubana Ivette García, el tiempo de la región "no tienen por qué coincidir - necesariamente- con el de la historia nacional, tiene su propio ritmo, su dualidad de carácter (objetivo-subjetivo) y resulta más acabado en la medida que el investigador tiene mayor dominio de su objeto de estudio" (2001:9). El tiempo regional, confirma Viales (2010:6) es diferenciado respecto al tiempo nacional, porque existe una in (dependencia) de las realidades y de las subjetividades regionales y de las localidades.

En este encuentro geohistórico con énfasis económico y regional, el espacio y tiempo se diluyen, pierden la precisión que tradicionalmente habían tenido. Ahora sus límites se construyen en función de su articulación o integración en el espacio social -con una aproximada base geográfica inicial-. La precisión del tiempo en función de la construcción regional termina por consolidarse a partir de la regionalidad -sentido de pertenencia- o el regionalismo -política hegemónica homogeneizadora del centro de poder-. Si bien, estas líneas son imprecisas, es importante que puedan ser observadas con los ojos entreabiertos, porque si se pierden, también se esfuma la región. Un concepto que sintetiza muy bien los aportes que se han discutido sobre la región histórica lo hace la Dr. Iveth García González:

La región histórica es un espacio geográfico social -en este caso subnacional-, en el que confluyen características económicas, sociales, políticas, culturales e ideológicas que revelan una mismidad que la singulariza, sin que por ello deje de pertenecer al entramado exterior, nacional e internacional del que forma parte. La ciudad, en este contexto, actúa como centro jerarquizante, hacia y desde el cual fluye la civilización. (2000b:106)

La geografía y la historia, se convencieron a lo largo de su devenir que desde sus marcos convencionales solo podían captar fragmentos muy herméticos de la realidad, así toman a la región como instrumento heurístico para estudiar la complejidad de la realidad. Sin embargo, es la antropología quien parece haber comprendido pronto este dilema.

Antropología y región: El precursor más importante de los estudios regionales desde la antropología en México -nuestro referente más cercano- fue Manuel Gamio, quien diseñó en 1919 el Programa de Antropología para el mejoramiento de las poblaciones regionales de la república (Fábregas, 2010:43). Para Gamio, la región es “el territorio de una población cultural y socialmente homogénea, con una historia compartida que puede distinguirse de otras", visto 
así, el estudio de la región, no es el estudio de espacio físico, sino de la gente, de tal manera que "una frontera se crea cuando se ponen en contacto sociedades con ecologías culturales diferentes y comienzan una interrelación que, a su vez, resultará en una sociedad particular" Fábregas (2010:160).

El reconocimiento de estas estructuras complejas regionales solo puede ser distinguidos según Stewart (1955) citado por Boehm (1997) a partir de los proceso simultáneos de integración $y$ articulación -propuestas con anterioridad por los geógrafos Bassol Batalla y Bataillon respectivamente-. Boehm menciona como elementos horizontales las clases o las agencias estatales, eclesiásticas, financieras, mercantiles, etc., y por elementos verticales el parentesco, las lealtades, la clientela, el mercado, los santuarios y otros; estos últimos, como propios del ser regional, es decir, de la regionalidad.

Sin embargo, no se pretende sumar los tres enfoques propuestos (geografía, historia y antropología), sino entender desde la perspectiva de cada uno, sus aportaciones y limitantes, pero dejar claro sobre todo, de la necesidad inter y transdisciplinaria de la ciencias para aproximarnos a la región, su complejidad social y cultural. La región se convierte entonces en un elemento heurístico para cada una de las disciplinas que por sí solas son insuficientes para descifrar la totalidad y complejidad regional.

\section{Las regiones de la meseta caraceña}

En el pacífico sur de Nicaragua se ubica el departamento de Carazo con una extensión de $1,032 \mathrm{Km}^{2}$, y repartido política y administrativamente en ocho municipios: Jinotepe, Diriamba, San Marcos, Dolores, El Rosario, Santa Teresa, La Paz de Carazo y La Conquista, siendo Jinotepe, la cabecera departamental y por lo tanto, el centro político o alcaldía mayor, donde se ubican las instituciones estatales y financieras más importantes del departamento. Sin embargo, la meseta caraceña vista con un enfoque regional se reconfigura en un entramado geográfico, ecológico y cultural, que da origen a cuatro regiones históricas: 1) Triángulo de oro del café, 2) Las comunidades pesqueras de la meseta, 3) La región de las comunidades rurales y 4) La región de los pueblos del sur.

\section{Región del Triángulo de oro del café}

La meseta caraceña ${ }^{2}$ como región, se eleva entre los 620 y 500 metros de altura sobre el nivel del mar, su parte alta es una planicie que se inclina suavemente de noreste a sureste, con una pendiente de 0 a $2 \%$, aunque en algunos lugares alcanza hasta $8 \%$. A esta región se denominó como Triángulo de oro del café durante el auge cafetalero en las postrimerías del siglo 2. La meseta Caraceña es una formación geológica (Meseta), sobre la cual se asienta el departamento de Carazo. Ésta comprende tres niveles de altura: parte alta $(500$ a $620 \mathrm{msnm})$, parte media (200 a $500 \mathrm{msnm}$ ) y parte baja (0 a $200 \mathrm{msnm}$ ). Sobre ella, hablando desde el punto de vista político-administrativo, se ubican ocho municipios, tres de ellos abarcan los tres niveles de altitud (Jinotepe, Diriamba y Santa Teresa), el resto se ubican distribuidos entre a parte alta y la parte media de la meseta. 
XIX e inicios del siglo XX. La parte noreste inicia con el fin de las serranías del Crucero al sur del departamento de Managua -la capital del país-, desde la comunidad La Danta, y se prolonga en dos direcciones; por el sureste, discurre hasta alcanzar los municipios de Santa Teresa y La Paz de Carazo, donde se pierde levemente hasta llegar al departamento de Rivas; y por el occidente, llega hasta la comunidad Las Esquinas y se enrumba hacia el departamento de Masaya, bordeando a todo el municipio de San Marcos.

El Triángulo de Oro del café en la meseta caraceña, es la región más densamente habitada, con el 61\% de la población departamental (AMUC, 2002), pues comprende los centros urbanos de los municipios más grandes: Jinotepe, Diriamba y San Marcos, así como el municipio de Dolores, que es el más pequeño y el más densamente poblado. Estas ciudades dibujan el perímetro de un centro verde, habitado por comunidades internas semirurales, donde se practica la caficultura de sombra, la agricultura de subsistencia por campesinos sin tierra o cooperados, además de la pequeña industria, que contrasta con los parques industriales textiles que se encuentran entre las ciudades, canalizando la mano de obra -en su mayoría femenina- pero además, explotando el acuífero que posee la región, que abastece al 80\% de la población del departamento.

La urbanización del Triángulo de oro solo pudo despegar con el auge cafetalero, cuando la región de oriente de Nicaragua ${ }^{3}$ tenía como centro jerarquizante a la ciudad de Granada que paulatinamente fue perdiendo su hegemonía sobre los pueblos de Jinotepe y Diriamba, cuando éstos cambian de categoría por sus propias características socioeconómicas, urbanísticas y políticas. Así, el 11 de febrero de 1883, el Senado y Cámara de la República de Nicaragua acuerdan elevar a la villa de Jinotepe a la categoría de ciudad y al pueblo de Diriamba al rango de Villa, que hasta en 1894 alcanza el rango de ciudad bajo la presidencia del general José Santos Zelaya, mismo que crea el departamento de Carazo en 1891. No es, sino hasta el 18 de febrero de 1916 por gestiones del senador caraceño Anastasio Somoza Reyes que se eleva a la villa de San Marcos a la categoría de ciudad, constituyéndose en la tercera ciudad de importancia en la región ${ }^{4}$. San Marcos, a diferencia de las dos primeras no es un pueblo originario, por el contrario, sus primeros pobladores, al parecer de Guerrero y Soriano (1964:69) fueron vecinos de la ciudad de Jinotepe y Nindirí que poblaron el territorio, ubicándose inicialmente en las inmediaciones de las pilas de Sapasmapa o en el asiento actual del barrio La Cruz, por la facilidad de obtener agua potable.

3. Así se denominaba al Pacífico sur de Nicaragua durante el siglo XIX, hasta que en el siglo XX se divide en dos regiones; la del Pacifico sur, conformada por los departamentos de Granada, Masaya y Carazo y la región del Pacífico Sur que comprende el departamento de Rivas.

4. Inferencia construida a partir de los datos tomados del compendio de gacetas que presenta en sus estudios el periodista e historiador local, Manuel Meza Fernández (2002), el matrimonio de historiadores Guerrero y Soriano (1964), y de los datos aportados por los tesistas Ingrand y Esteban (2013) que realizan un estudio de la evolución urbana del municipio de Jinotepe. 
Hoy la región está dominada por el comercio, que tiene como polos principales los mercados $^{5}$ de Jinotepe y Diriamba, siendo el primero de carácter regional, donde convergen productores, comerciantes y consumidores de los departamentos de Managua, Granada, Rivas y Masaya, sobre todo de éste último. Buses, camiones, cabezales, descargan mercadería para abastecer el mercado jinotepino, donde los precios de los productos importados como la ropa nueva o usada, electrodomésticos y otros, son los más competitivos, no así los granos básicos, abarrotería o verduras que son más favorables en Diriamba, ya que los productores locales de la región y las comunidades rurales aledañas comercializan su producción en este mercado, debido a que los caminos internos de la región llegan directamente hasta el centro de compras. Al recorrer las calles de los centros urbanos en la región es común observar rótulos de negocios locales o marcas internacionales, en especial las comidas rápidas, por ejemplo un rótulo de la TIP-TOP, que obstruye la vista del volcán Mombacho (Granada) desde la entrada de Jinotepe cuando se viene desde Diriamba. Las casas son utilizadas como negocios particulares, de personas que las alquilan y las modificaron en módulos para exhibir los productos ${ }^{6}$.

\section{Región de las comunidades pesqueras de la meseta caraceña}

Esta región tiene su base en la zona costera de la meseta caraceña, se extiende a lo largo de cincuenta kilómetros del litoral Pacífico de noreste a sureste. Inicia en el estero de Tecolapa -que sirve de límite con Managua- hasta la bocana de Escalante o mar Chorotega ${ }^{7}$-límite con el departamento de Rivas-. La región se reparten entre los municipios de Diriamba, Jinotepe y Santa Teresa, esto incluye la faja costera y la llanura exterior que se interna hasta $10 \mathrm{~km}$ tierra adentro, hasta alcanzar los 200 msnm aproximadamente. El clima es cálido y muy seco, caen entre 800 a $1200 \mathrm{~mm}$ de agua anual con períodos caniculares prolongados (CENAGRO 2013; AMUC, 2002). En la parte occidental de esta región de las comunidades pesqueras se encuentran, entre otras, las comunidades mayormente pobladas: La Boquita, Casares, Huehuete y Tupilapa, que son parte de los municipios de Diriamba y Jinotepe. En la parte oriental que le corresponde a Santa Teresa.

La pesca es la actividad económica por excelencia de esta región. Cerca del $80 \%$ de la población vive de ella, directa o indirectamente; no solo van al mar a pescar, también arman o desenredan las redes, componen las lanchas, limpian y venden pescado, etc. Los pescadores tienen el mar para ellos, pero no son dueños de los medios de producción, sino que son financiados por los acopios pesqueros de la región, expresa Erling (comunicación personal, 2008) -líder comunitario de Huehuete- que estos últimos proveen a cada grupo de pescadores de insumos 5. Entendido en este caso como sitio público destinado para vender, comprar o permutar bienes y servicios.

6. Según el Censo Económico Urbano de Carazo (2010:15), existen 4,290 viviendas con actividad económica, muy por arriba de las edificaciones propias para negocio que suman 1, 146 y más alejado de los mercados, que a pesar de alta concentración en especial en Jinotepe y Diriamba suman 921.

7. Llamado así por Dávila Bolaños, al referirse a las ceremonias y ritos que realizaban los mangues entre el 9 y 28 de abril en las costas de la mar, entre Masachapa y la desembocadura de rio Escalante. 
con un valor de cien dólares aproximadamente y después de la jornada de trabajo, estos tienen que pagar en producto el valor del insumo o lo que hayan consumido de él, el resto del producto es pagado a los pescadores por los acopios a precios que ellos establecen. En cada lancha (del acopio o dueños particulares) se montan entre cinco y seis pescadores equipados con una batería de doce voltios, doce galones de gasolina, un GPS, un quintal de hielo, dos cajas de caballón ${ }^{8}$, un trasmallo, redes y anzuelos, así como un galón de agua, comida, cigarros y capote por si llueve. Luego de la jornada se debe pagar a los inversores. La ganancia es repartida entre los pescadores que van y el propietario de la lancha, por ejemplo si entran cinco pescadores al mar, la ganancia se divide entre seis, el seis es la lancha o sea el dueño de la lancha. Cuando no hay ingreso, se anota a los pescadores como deuda según lo que hayan utilizado de los insumos. En las pérdidas no es anotado el dueño de la lancha.

Los acopiadores se encargan de vender el producto a empresas de la capital. Hace algunos años los distribuían ellos, ahora llegan los camiones de las empresas con sus contenedores porque la demanda del producto ha crecido. También llegan comerciantes desde los mercados de Jinotepe y Diriamba o pobladores que bajan en sus vehículos particulares a comprar mariscos frescos, especialmente los sábados por la madrugada. Pero no todos viven de la pesca. Se pueden ver pequeños emprendimientos: comedores, bares, billares, galleras y pulperías. Hay quienes trabajan como meseros o cocineras en algunos restaurantes y hoteles, o como doméstica en las quintas privadas. También se dedican la crianza de cerdos, aves de corral, producción de artesanías como prendas o adornos con recursos del medio (conchas, arena, astillas), que venden a visitantes que llegan a los pocos restaurantes que se han instalado en la región, principalmente en el centro Turístico La Boquita que es propiedad de INTUR (Instituto Nicaragüense de Turismo).

\section{La región de las comunidades rurales}

En esta región se incluye la parte norte-alta del municipio de San Marcos, la zona media de Diriamba y Jinotepe, el sur de Santa Teresa y la totalidad del municipio La Conquista9 ${ }^{9}$ A pesar de que los otros municipios -Dolores, El Rosario y La Paz poseen áreas para cultivo (granos básicos y caña de azúcar), no se incluyen por su dimensión territorial, su ubicación en la parte alta de la meseta y las relaciones sociales que se construyen alrededor de estos pueblos.

Las comunidades rurales, a veces tienen forma de caseríos dispersos y otras de caseríos concentrados. En ambos casos, constituyen núcleos sociales con características muy similares; bajos porcentajes de alfabetismo ${ }^{10}$, niveles altos de pobreza y desempleo. Aunque el censo de

8. Es un pez de importación, no apto para el consumo humano. Vienen recubiertos de un aceite especial que atrae a las presas. Cada caja tiene un valor aproximado de 18 dólares.

9. Según la caracterización socioeconómica del municipio, el $90 \%$ de su población es rural, tanto como su territorio. 10. En el 2005, la tasa de analfabetismo según el censo de ese año, era, en la zona rural de Carazo de $20.2 \%$, más alta que el promedio nacional rural que era de 18.5\%. Sin embargo se debe recordar que para 2005, habían 
2005 muestra una fuerte tendencia a trabajar en la misma localidad ${ }^{11}$, no es la realidad de las comunidades rurales en la actualidad, pues sus habitantes, generalmente los jóvenes buscan en la ciudad otras alternativas de empleo, que no sea el trabajo en el campo. Los destinos más buscado por los jóvenes son Diriamba o la capital, Managua, donde se someten a cualquier trabajo. Algunos también se quedan en las comunidades cercanas para de allí movilizarse hacia alguna zona franca de la región.

La vida cotidiana en estas comunidades por lo general comienza a las cinco o cuatro de la madrugada, los roles son muy marcados entre las mujeres y los hombres: la mujer se levanta a arder el fuego para preparar el café, barre el patio y ordena la casa. El hombre por su parte se levanta a ver y alimentar a los animales y en el caso de tener vacas, las ordeñan para luego llevarlas a pastar, baja al rio a bañarse y sube agua ${ }^{12}$ para las labores domésticas -. Respecto a la religión, es en estas comunidades rurales donde más se ha desarrollado el protestantismo en sus diferentes denominaciones ${ }^{13}$. En cada comunidad existe una o hasta tres células de hermanos protestantes, que se reúnen cada tarde o noche para realizar sus cultos o vigilias.

Para finalizar sobre esta región de las comunidades rurales se debe mencionar que si bien tienen muchos rasgos comunes también son diferentes en sí mismas y pueden identificarse dos tipos de comunidades; las comunidades deprimidas, que se ubican sobre suelos con poca o ninguna fertilidad, zonas de difícil acceso y por lo tanto carecen de la mayoría de los servicios básicos, por ejemplo Aguas Calientes y la Pita. Y comunidades intermedias, que sirven de puente entre las comunidades deprimidas y las ciudades o cabeceras municipales; en estas comunidades suele haber movimiento comercial y puestos de intermediaria, ejemplo claros son; la zona urbana del municipio La Conquista u otras comunidades como La Trinidad en Diriamba.

pasado 15 años de gobierno neoliberal en Nicaragua, que promovió políticas de "Autonomía" (especialmente administrativa) dejando la responsabilidad de la educación nacional en la empresa privada que limitó el acceso a la educación, violentando un derecho humano fundamental, que aumentó las cifras de analfabetismo que se habían bajado con la revolución de los años 80, cuando se dio la Gran Cruzada Nacional de Alfabetización. En la actualidad el gobierno Sandinista se ha mantenido desde 2007 -como una continuidad de la Revolución Popular Sandinistaha declarado a Carazo como territorio libre de analfabetismo, meta lograda a través de la alfabetización como modalidad de graduación para el bachillerato de estudiantes de secundaria, sin embargo la lucha actual es la batalla por el sexto grado y la matricula en la educación inicial.

11. El rango de las personas que trabajan en el mismo municipio anda entre los $60 \%$ y $75 \%$, a excepción de Dolores.

12. Esta práctica de subir agua ha disminuido con la instalación de pozos, por ejemplo en La Conquista -que es esencialmente rural- para el 2005 el abastecimiento de agua por pozo era de $39.5 \%$, cuando el promedio nacional era de 16.4 según el último censo nacional realizado.

13. Según el último censo nacional, para 1995 la población del departamento de Carazo, creyente en la fe católica representaba el $74 \%$, porcentaje que bajó hasta el $56.8 \%$ para el años 2005, mientras que los que declararon pertenecer a la religión evangélica aumentan en el mismo periodo de un $17.5 \%$ a un $25.2 \%$. si bien este dato se refiere al departamento en general, se debe recordar que esta región de comunidades rurales representa la mayor parte del departamento y aunque no es la más poblado, si es la menos atendida por la iglesia católica por la cantidad de sacerdotes que tiene el departamento, que generalmente es uno o dos por municipio, mientras la iglesia evangélica tiene hasta tres pastores o pastoras en cada comunidad rural. 


\section{La región de los pueblos del sur}

Esta región se ubica en la parte oriental de la meseta Caraceña, con una extensión territorial aproximada de $42 \mathrm{Km}^{2}$, si tomamos en cuenta que está formada por los centros urbanos y periféricos de los municipios de El Rosario, La Paz de Carazo y solamente la parte alta o urbana de Santa Teresa, ya que su ruralidad fue abarcada en la región de Las comunidades rurales. La topografía de esta región es completamente plana especialmente para el municipio de El Rosario con declive de 15\%, algunas ondulaciones moderadas se encuentran en La Paz de Carazo y la periferia de Santa Teresa que van de $4 \%$ a 50\%, formando pequeñas cañadas que albergan los reductos de bosque primario o secundario (medianos-altos, latifoliado-perennifolios). Sin embargo, no se presentan accidentes orográficos ni hidrográficos de importancia, incluso para Santa Teresa en la parte alta.

Los datos censales de 2005 indican que son los hombres los que se encargan del campo y la industria, mientras que la mayoría de las mujeres de esta región se dedican a las actividades de servicio con excepción de El Rosario. Un ejemplo claro de esto son los trapiches (actividad que data de la época colonial en Nicaragua), que además son el mayor vínculo entre esta región y la región de Las comunidades rurales, para la compra de su materia prima, pero a la vez, esta actividad los vincula con otras regiones, no solo de Carazo, sino también de Nicaragua. En esta región existen cerca de 50 trapiches y son movidos con motor estacionario en cada plantel y no con bueyes como se hacían anteriormente [comunicación personal con Roberto Conrado, 2018].

\section{DISCUSIÓN}

Desde el punto de vista de la geografía tradicional que describe Espejo Marín (2003), Carazo se ubica sobre una meseta que tiene tres niveles de altura con características particulares: clima, humedad, suelos, etc. Las formas de explotación de sus recursos dependen de los mismos recursos, es decir, de la adaptación humana a su entorno. Sin embargo, esta adaptación no es irracional o simplemente intuitiva como lo sería para otras especies vivas. El hombre, interviene con un factor cultural (Steward, 1955), es decir, con un conocimiento de técnicas, instrumentos, procedimientos, etc., que comparte con otros seres humanos de otros lugares, pero además con un conjunto de relaciones, que no se limitan al espacio territorial sino que se inserta en un entramado social y económico más amplio que vincula cada región con las otras. Si la base de su producción nace de la adaptación de su entorno, la comercialización de sus productos sobrepasa la región, es decir comprende un sistema de articulación, tal como las redes complejas que propone Boehm (2007).

El otro punto es que la región geográfica no determina la actividad del hombre sobre el medio, lo cual es evidente en la parte alta de la meseta, sobre la cual hemos distinguido dos regiones; el triángulo de oro y los pueblos del sur. Ambos tienen condiciones ambientales análogas 
y por lo tanto, tendrían que tener sistemas de producción semejantes. Sin embargo, su diferencia radica en su historia y cultura; mientras, las ciudades del triángulo de oro fueron nacidas de pueblos originarios, las otras fueron creadas en la colonia como un proceso de redistribución de las poblaciones. En consecuencia, sus prácticas culturales de consumo y de relaciones tendrán que ser distintas. En otras palabras, no fueron las condiciones ambientales que definieron la cultura de estas regiones, sino su historia, su tiempo regional que no coincide necesariamente con el tiempo nacional tal como lo proponen García González (2000b) y Viales (2010).

Las fronteras entre cada una de las regiones, no concuerda con los limites políticos, pero tampoco son tan distinguibles como éstos porque son linderos sociales, tal como lo planteas Fábregas (2010:160) desde la antropología social "una frontera se crea cuando se ponen en contacto sociedades con ecologías culturales diferentes y comienzan una interrelación que, a su vez, resultará en una sociedad particular”.

\section{CONCLUSIONES}

Desde el punto de vista conceptual, podemos concluir con una aproximación del concepto región histórica como un espacio social dialéctico y dinámico, vivido en función de determinadas necesidades y construido en función de determinados intereses, la elasticidad de sus linderos (intra, inter y transnacional) no se corresponde necesariamente con las divisiones políticas administrativas, y aunque su desarrollo histórico obedece a su propia dinámica, no deja de estar ligado e influenciado por uno o más centros de poder regional, que a su vez obedecen a un poder central: El Estado. Los cambios socio-económicos, políticos, culturales e incluso ecológicos, por su carácter antropocéntrico, suceden cada vez más rápido, lo que forja una especie de identidad en movimiento por la interrelación entre la cultura y el medio ambiente próximo, teniendo al hombre (sujeto político) como parte integral del proceso. Lo más importante de la región histórica es que es un espacio vivido y que esa vida en común está en la conciencia de quienes lo han vivido.

Desde el punto de vista empírico asumimos que las cuatro regiones que se identificaron en Carazo, tienen una dinámica interna, asociada a sus características ecológicas y productivas, que configuran sus relaciones de trabajo y por lo tanto su cultura. Sin embargo, existe una relación dinámica entre las regiones que están regidas por el comercio, las relaciones sociales y la dinámica cultural. Los límites regionales (que son construcciones sociales) no coinciden con los límites políticos y administrativos, pero tampoco los niegan ni son un obstáculo para la dinámica regional. Sin embargo, sería interesante para los gobiernos municipales que se plantearan esta forma de ver y administrar el territorio porque podrían dar soluciones conjuntas e integrales a las poblaciones regionales, reduciendo la brecha entre la ciudad y la periferia. 


\section{BIBLIOGRAFÍA}

AMUC-GIZ (2012). Atlas departamental Carazo. Carazo, Nicaragua. Fondos del proyecto.

Boehm, B. (1997). El enfoque regional y los estudios regionales en México: geografía, historia y antropología. Relaciones 72, Vol. 18, pp. 17-46.

Conrado, R. (11 de enero de 2018). Los pueblos del sur. Trabajo: trapiches. (W. Guevara, entrevistador)

Espejo, M. (2003). Anotaciones en torno al concepto de región. NIMBUS, N 11-12, pp. 67-87. ISSN: 1139-7136

Fábregas, P. (2010). Configuraciones regionales mexicanas. Un planteamiento antropológico. México. Tomo 1. Ed. El Aleph Digital, S.A de C.V.

García González, I. (2000a). Experiencias obtenidas a partir del estudio de una región cubana deprimida. Instituto de Historia de Cuba (paper).

García González, I. (2000b). Vivir la frontera imperial; Baracoa, la primada de Cuba. RMC, N 9, pp. 104-139. DOI: 10.22403/ UQROOMX/RMC09/03

Guerrero, J. \& Soriano, L. (1964). Monografía del departamento de Carazo. En Colección Nicaragua: El estudio monográfico de cada uno de los departamentos de la República. Managua, Nicaragua. Fondos propios.
INIDE (2005). Caracterización Sociodemográfica del Departamento de Carazo. En VIII Censo de población y IV de vivienda 2005. Managua, Nicaragua. Ed. Fondos propios.

INIDE (2010). Censo urbano Carazo. Recuperado de http://bit.ly/2HtQAaO

INIDE-MAGFOR (2013). IV Censo Nacional Agropecuario (CENAGRO). Managua, Nicaragua. Tomo 2: Departamento de Carazo. Ed. Fondos propios. Recuperado de Ley de municipios (Ley 40)

Molina, M. (1986). Paisaje y región: una aproximación conceptual y metodológica. En García, A. (Coord.): Teoría y práctica de la geografía, Madrid, Alhambra, pp. 63-87.

Pérez, E. (10 de enero de 2018). Las comunidades pesqueras de Carazo. Vida cotidiana. (W. Guevara, entrevistador)

Steward, J.H. (1955). Theory of Culture Change: The Methodology of Multilinear Evolution. Urbana.

Viales, R. (2010). La región como construcción social, espacial, política, histórica y subjetiva. Hacia un modelo conceptual/ relacional de historia regional en América Latina. Geopolítica, Vol. 1 (1), pp. 157172. ISSN: 2172-3958. 